\title{
Neumatosis quística intestinal y peritoneal, causa de neumoperitoneo Revisión de la literatura a propósito de un caso
}

\author{
Intestinal and peritoneal cystic pneumatosis as a cause of \\ pneumoperitoneum. Review of the literature about a case
}

\author{
Yeni Arroyave ${ }^{1}$ Germán Ruiz², María del Mar Meza³ ${ }^{3}$ Edwin Muñoz ${ }^{4}$, Wilson Muñoz ${ }^{5}$ \\ Médica, residente del programa de Cirugía, Universidad del Cauca, Popayán, Colombia \\ Médico, especialista en Radiología; profesor, Departamento de Medicina Interna, Universidad del Cauca, Popayán, Colombia \\ Médica, especialista en Cirugía General; docente, Departamento de Cirugía, Universidad del Cauca, Popayán-Colombia. \\ Médico, especialista en Cirugía General, Universidad del Cauca, Popayán, Colombia \\ Médico, especialista en Cirugía Vascular y Angiología; docente, Departamento de Cirugía, Universidad del Cauca, Popayán, \\ Colombia
}

\section{Resumen}

Introducción. La neumatosis intestinal es una condición rara que se caracteriza por la infiltración submucosa o subserosa de gas en el tubo digestivo. Se encuentra más frecuentemente en el intestino delgado y, pocas veces, en localización extraintestinal. Su prevalencia estimada es de 0,03\%.

Métodos. Se hizo una búsqueda bibliográfica en las bases de datos biomédicas Medline Pubmed, Science Direct, Tripdatabase y Uptodate, usando como palabras clave 'neumoperitoneo' y 'neumatosis cistoide intestinal' Para la presentación del caso clínico, se tomaron datos de la historia clínica y, además, imágenes de radiografia, tomografías y material fotográfico del procedimiento quirúrgico.

Presentación del caso. Se trata de una mujer de 63 años de edad con antecedentes de colagenopatía, que consultó por dolor y distensión abdominal. Aunque no tenía signos de irritación peritoneal, las imágenes diagnósticas revelaron neumoperitoneo y líquido libre en la cavidad peritoneal, por lo que se sospechó una perforación intestinal y se practicó una laparotomía exploratoria, en la cual se encontró neumatosis quística intestinal y peritoneal.

Conclusiones. No todos los casos de neumoperitoneo requieren cirugía. Existen casos espontáneos y sin irritación peritoneal, secundarios a neumatosis quística intestinal. En los casos de neumoperitoneo en pacientes estables con un diagnostico etiológico no muy claro, y en quienes se desee descartar perforación intestinal o

Fecha de recibido: 20/02/2019 - Fecha aceptación: 3/10/2019

Correspondencia: Yeni Arroyave, Carrera 6 № 10 Norte-143, Hospital Universitario San José, Departamento de Cirugía, tercer piso, Popayán, Colombia. Teléfono: (301) 263-2778

Correo electrónico: yarroyave@unicauca.edu.co.

Citar como: Arroyave Y, Ruiz G, Meza M del M, Muñoz E, Muñoz W. Neumatosis quística intestinal y peritoneal, causa de neumoperitoneo. Revisión de la literatura a propósito de un caso. Rev Colomb Cir. 2020;35:93-9. https://doi.org/10.30944/20117582.592

Este es un artículo de acceso abierto bajo una Licencia Creative Commons - BY-NC-ND https://creativecommons.org/licenses/by-nc$\mathrm{nd} / 4.0 /$ deed.es 
isquemia mediante exploración quirúrgica, la laparoscopia diagnóstica es una buena opción y menos agresiva que la laparotomía.

Palabras clave: tracto gastrointestinal; intestino delgado; neumatosis cistoide intestinal; neumoperitoneo; tratamiento conservador.

\begin{abstract}
Introduction: Intestinal pneumatosis is a rare condition that is characterized by submucosal or subserosal gas infiltration in the digestive tract. It is found more frequently in the small intestine, and rarely in an extraintestinal location. Its estimated prevalence is $0.03 \%$.

Methods: A literature search was performed in the Medline Pubmed, Sciencedirect, Tripdatabase and Uptodate databases, using as keywords Neumoperitoneum and Intestinal Cystoid Neumatosis. For the presentation of the clinical case, medical history data were collected, in addition to radiography images, tomography and photographic material of the surgical procedure.

Clinical case: This is a 63-year-old woman with a history of collagenopathy, who consulted for abdominal pain and distension. Although she had no signs of peritoneal irritation, diagnostic images revealed pneumoperitoneum and free fluid in the peritoneal cavity. Intestinal perforation was suspected, and an exploratory laparotomy was performed, in which intestinal and peritoneal cystic pneumatosis in was found.

Conclusions: Not all cases of pneumoperitoneum require surgery. There are spontaneous cases without peritoneal irritation, secondary to intestinal cystic pneumatosis. In cases of pneumoperitoneum in stable patients, with a not very clear etiological diagnosis, and in those who wish to rule out intestinal perforation or ischemia by surgical exploration, diagnostic laparoscopy is a good option and less aggressive than laparotomy.
\end{abstract}

Keywords: gastrointestinal tract; intestine, small; pneumatosis cystoides intestinalis; pneumoperitoneum; conservative treatment.

\section{Introducción}

La neumatosis intestinal es una condición rara que se caracteriza por la infiltración submucosa o subserosa de gas en el tubo digestivo. Se encuentra más frecuentemente en el intestino delgado $y$, pocas veces, en localización extraintestinal ${ }^{1-4}$. Sus manifestaciones clínicas son inespecíficas y puede ser detectada por una variedad de imágenes diagnósticas.

El propósito de este artículo fue revisar los aspectos más relevantes sobre la etiología, la presentación clínica y el diagnóstico de esta condición poco común, además de presentar el caso de una paciente con neumoperitoneo espontáneo por neumatosis quística de localización intestinal y extraintestinal.

\section{Métodos}

Para la revisión del tema, se hizo una búsqueda bibliográfica en las bases de datos biomédicas Medline Pubmed, Sciencedirect, Tripdatabase y Uptodate, usando como palabras clave 'neumoperitoneo' y 'neumatosis cistoide intestinal'. Para la presentación del caso clínico, se tomaron datos de la historia clínica y, además, imágenes de radiografía, tomografía y material fotográfico del procedimiento quirúrgico.

\section{Revisión de la literatura}

\section{Definición}

La neumatosis intestinal se caracteriza por la infiltración submucosa o subserosa de aire en 
el tubo digestivo. Es un signo clínico, más que una enfermedad. Puede pasar de ser un hallazgo incidental a ser una afección potencialmente mortal ${ }^{\mathrm{I}}$.

\section{Epidemiología}

La neumatosis intestinal se puede localizar en cualquier parte del tubo gastrointestinal y lo hace especialmente en el intestino delgado ${ }^{3}$. La localización extraintestinal es poco común ${ }^{4}$. Se observa con mayor frecuencia en los primeros días de vida y después de los 60 años de edad, y su prevalencia estimada es de $0,03 \%^{2}$. Se presenta en ambos sexos, aunque es más frecuente en el masculino, con una relación de I,9 a 3,5 hombres por cada mujer ${ }^{5}$.

\section{Clasificación y etiología}

Existen dos tipos de neumatosis intestinal, una quística, con burbujas aisladas de aire en la pared intestinal, y la otra lineal, con aspecto de aire en banda. Esta última se asocia con obstrucción intestinal, vólvulo, invaginación intestinal, hemorragia o infarto intestinal en, el $90 \%$ de los casos, aproximadamente.

Según los factores desencadenantes implicados, se puede hablar de una forma primaria o idiopática ( $15 \%)$ y otra secundaria $(85 \%)$ relacionada con enfermedades abdominales y extraintestinales, como obstrucción y pseudoobstrucción intestinal, isquemia mesentérica, diverticulosis, inflamación o infección intestinal (enfermedad de Crohn, colitis ulcerosa, enterocolitis necrosante, gastritis enfisematosa de la diabetes, tiflitis o enfermedad ulcerosa péptica), enfermedad pulmonar (asma, enfermedad pulmonar obstructiva crónica, fibrosis quística o infecciones respiratorias), tumores o enfermedades del colágeno (esclerodermia, enfermedad mixta del tejido conjuntivo, lupus eritematoso sistémico), traumas, inmunodepresión y esteroides ${ }^{2-4,6,7}$.

\section{Patogénesis}

Se han propuesto numerosas hipótesis para explicar la patogénesis de la neumatosis intesti- nal, incluyendo causas mecánicas, bacterianas y bioquímicas; las más aceptadas son las teorías mecánica y bacteriana. Aunque las teorías son claramente diferentes, no son necesariamente excluyentes entre sí. Es probable que múltiples mecanismos patogénicos estén involucrados en la formación de la neumatosis intestinal ${ }^{8}$.

La teoría mecánica postula que el gas entra en la pared del intestino, bien sea de la superficie de la luz intestinal a través de rupturas en la mucosa o a través de la superficie serosa mediante el seguimiento a lo largo de los vasos sanguíneos mesentéricos; este gas puede extenderse a lo largo del mesenterio a sitios distantes.

La teoría bacteriana postula que las bacterias de la luz intestinal producen cantidades excesivas de gas hidrógeno por medio de la fermentación de hidratos de carbono y otros productos alimenticios. A medida que la presión del gas dentro de la luz intestinal aumenta, el gas puede ser forzado directamente a través de la mucosa y quedar atrapado dentro de la submucosa ${ }^{\mathrm{I}, 6,8}$. El sobrecrecimiento bacteriano se facilita en la esclerodermia por el estado hipóxico y la hipomotilidad del intestino de estos pacientes ${ }^{9}$.

\section{Manifestaciones clínicas}

Las manifestaciones clínicas son inespecíficas. Los pacientes suelen estar asintomáticos, pero cuando se presentan síntomas, pueden incluir emesis, distensión abdominal, pérdida de peso, dolor abdominal, diarrea, estreñimiento y sangrado rectal $2,4,8,10$.

\section{Diagnóstico}

La neumatosis intestinal puede ser detectada por una variedad de diferentes modalidades de imágenes radiográficas, incluyendo radiografías simples, estudios con contraste, tomografía computarizada (TC), ultrasonido y resonancia magnética ${ }^{8}$. Cuando se detecta en una radiografía simple, la TC debería ser la siguiente prueba con el fin de confirmar el diagnóstico; además, proporciona datos sobre otras enfermedades abdominales que pudieran ser una causa subyacente, como el vólvulo y la obstrucción intestinal ${ }^{4,8, \mathrm{II}}$. 
Los hallazgos característicos de la neumatosis intestinal en la TC incluyen colecciones circunferenciales de aire adyacentes a la luz del intestino que se localizan paralelas a la pared del intestino, pero sin niveles hidroaéreos ${ }^{8}$.

Uno de los signos patognomónicos de la neumatosis quística es el neumoperitoneo sin signos de irritación peritoneal producido por la ruptura de un quiste, que se produce hasta en un tercio de los pacientes ${ }^{7}$. Aunque este signo existió en la paciente que se presenta, la neumatosis intestinal no se consideró antes de la cirugía; ella se intervino con el diagnóstico de perforación intestinal y la neumatosis quística fue un hallazgo intraoperatorio.

\section{Tratamiento}

El manejo terapéutico de la neumatosis intestinal debe dirigirse a la causa subyacente, siempre que sea posible. Sin embargo, es fundamental establecer si el paciente presenta signos clínicos que indiquen riesgo para la vida, como dolor abdominal grave o irritación peritoneal y, en la tomografía, estriación de la grasa, espacios quísticos con distribución vascular, líquido libre o neumatosis de la vena porta, casos en los cuales se debe considerar la intervención quirúrgica precoz $^{\mathrm{I}}$.

Los pacientes asintomáticos no precisan tratamiento y, en las formas leves de neumatosis intestinal, se recomienda el manejo conservador que incluye esteroides, dieta elemental, oxígeno hiperbárico y antibióticos. Como tratamiento antibiótico comúnmente se usa el metronidazol, en dosis de $500 \mathrm{mg}$, tres veces al día. Se cree que con este esquema terapéutico se erradican las bacterias formadoras de gas en la neumatosis intestinal. La administración de oxígeno hiperbárico se fundamenta en que es un gas tóxico para las bacterias intestinales anaerobias que contribuyen a la formación de aire dentro de los quistes, además de que el contenido de los quistes es principalmente gas no oxigenado. El suministro de altas concentraciones de oxígeno aumenta la presión parcial de oxígeno en la sangre venosa, mientras que disminuye la presión parcial de los gases no oxigenados, creando así un gradiente de difusión a través de la pared del quiste y obligando al gas atrapado a salir de los quistes ${ }^{13}$.

Como se mencionó anteriormente, la intervención quirúrgica se reserva para los casos en que hay amenaza de muerte, los cuales presentan síntomas graves o complicaciones como vólvulo o hemorragia intraabdominal, o si falla el tratamiento médico. Cuando los beneficios superan a los riesgos, se recomienda una laparoscopia diagnóstica, la cual puede ser, además de diagnóstica, terapéutica ${ }^{6,7,7,1,14}$.

\section{Caso clínico}

Se trata de una mujer de 63 años de edad, originaria de Cartagena, en la Costa Atlántica colombiana, residente en Popayán (Cauca) desde 6 meses antes del ingreso hospitalario. Consultó por un cuadro clínico de seis meses de evolución de distensión abdominal, dolor abdominal difuso, sensación de plenitud gástrica y, en ocasiones, emesis posprandial, pérdida de $20 \mathrm{~kg}$ de peso, aproximadamente, astenia, adinamia y, en el último mes, diarrea intermitente.

Sus antecedentes médicos incluían lupus eritematoso sistémico y esclerodermia diagnosticada en el 200I y sin tratamiento desde I8 meses, aproximadamente, antes del ingreso; además, esófago de Barret y reflujo gastroesofágico. No tenía antecedentes alérgicos ni farmacológicos, solo uno quirúrgico de histerectomía abdominal por miomatosis uterina 20 años atrás.

En el examen físico se encontró caquéctica, con tensión arterial sistémica de $97 / 70 \mathrm{~mm} \mathrm{Hg}$, frecuencia cardiaca de 87 latidos por minuto, frecuencia respiratoria de 16 respiraciones por minuto y temperatura de $36^{\circ} \mathrm{C}$. El abdomen estaba distendido, no doloroso a la palpación, sin signos de irritación peritoneal, timpánico a la percusión y con aumento del peristaltismo.

En los exámenes de laboratorio se encontró función renal y hepática normales, no había leucocitosis ni neutrofilia, la albúmina sérica fue de $3,5 \mathrm{~g} / \mathrm{dl}$ y el VIH fue negativo. En las radiografías de tórax y de abdomen en posición vertical, se 
observó una gran cámara de neumoperitoneo que desplazaba el hígado y el bazo hacia abajo.

Al no encontrarse signos de irritación peritoneal, se practicó una tomografía toraco-abdominal en la que se observó: derrame pleural bilateral con engrosamiento de las cisuras, bronquiectasias basales bilaterales, atelectasia parcial de ambos lóbulos inferiores, notoria distensión del esófago con sonda en su interior, hidroneumoperitoneo, abundante líquido libre en la cavidad abdominal y distensión de las asas intestinales (figuras I-3).

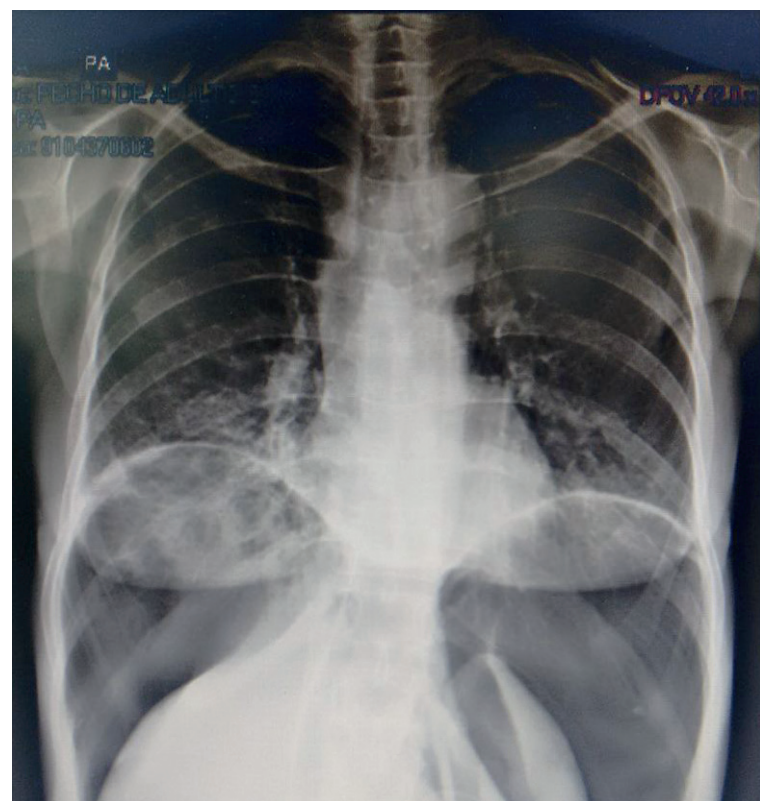

Figura 1. Radiografía de tórax en la que se observa imagen de neumoperitoneo.
La distensión abdominal mejoró parcialmente con la colocación de una sonda nasogástrica. Sin embargo, por los hallazgos de imagenología, de aire y líquido libre en la cavidad peritoneal, se sospechó perforación intestinal y se inició manejo antibiótico con ampicilina-sulbactam; además, se practicó una laparotomía exploratoria y se encontraron cerca de $700 \mathrm{ml}$ de ascitis y múltiples quistes neumáticos en el peritoneo parietal, predominantemente en el ligamento falciforme del hígado, y algunos en asas intestinales delgadas. También, se encontraron zonas ovoides de serosa despulida en las asas intestinales delgadas, lo cual sugería ruptura espontánea

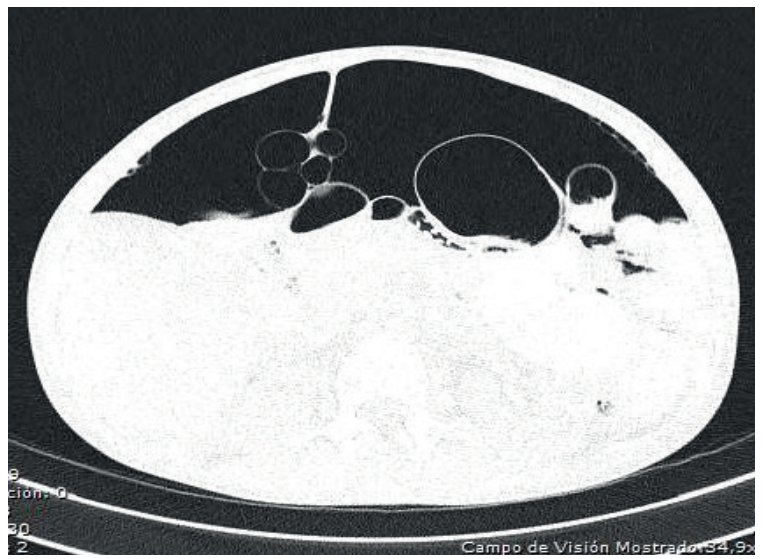

Figura 3. Tomografía computarizada, ventana pulmonar, donde se observa cámara de neumoperitoneo, además de gas que se distribuye en forma de burbujas en la pared del intestino delgado y en el ligamento falciforme del hígado.

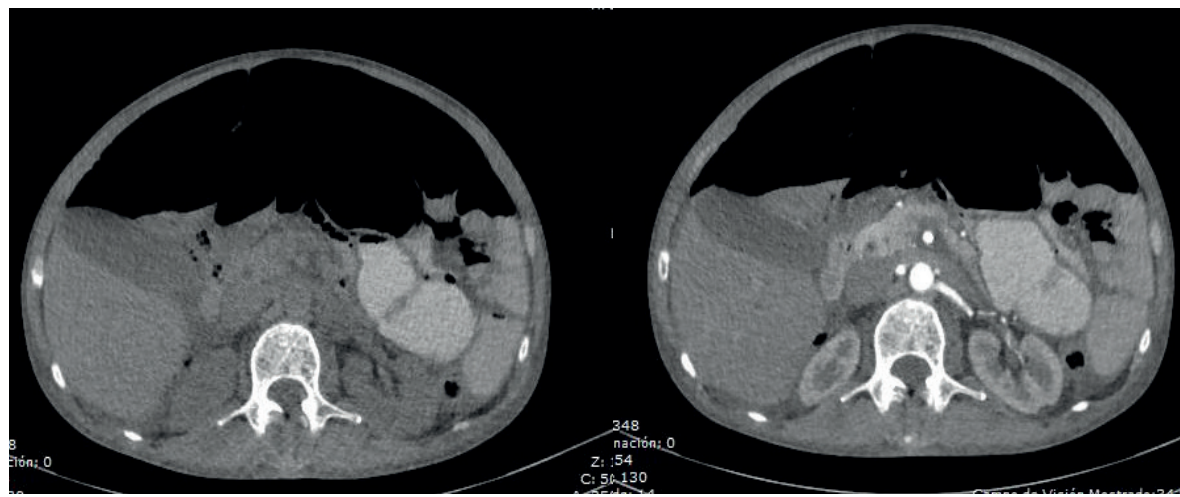

Figura 2. Tomografía computarizada de abdomen simple y con contraste, en las que se observa neumoperitoneo y líquido libre en la cavidad peritoneal. 
de quistes neumáticos intestinales (figuras 4-6). Con estos hallazgos, se hizo el diagnóstico de neumatosis quística intestinal y peritoneal como causa del neumoperitoneo espontáneo en esta paciente.

La paciente evolucionó adecuadamente en el periodo posoperatorio. El estudio citoquímico del líquido ascítico correspondió a un transudado, y la coloración de Gram y los cultivos fueron negativos. El estudio histopatológico reportó un quiste simple. Por la acentuada pérdida de peso, se practicó una endoscopia, la cual descartó neoplasia maligna en las vías digestivas altas.

Según la revisión bibliográfica, se consideró que el principal factor determinante del desarrollo de neumatosis intestinal fue la esclerodermia y el lupus eritematoso sistémico sin tratamiento, por lo que se remitió a reumatología para iniciar el tratamiento de sus enfermedades de base. Finalmente, la paciente egresó sin dolor ni distensión abdominal y con una adecuada tolerancia a la dieta.
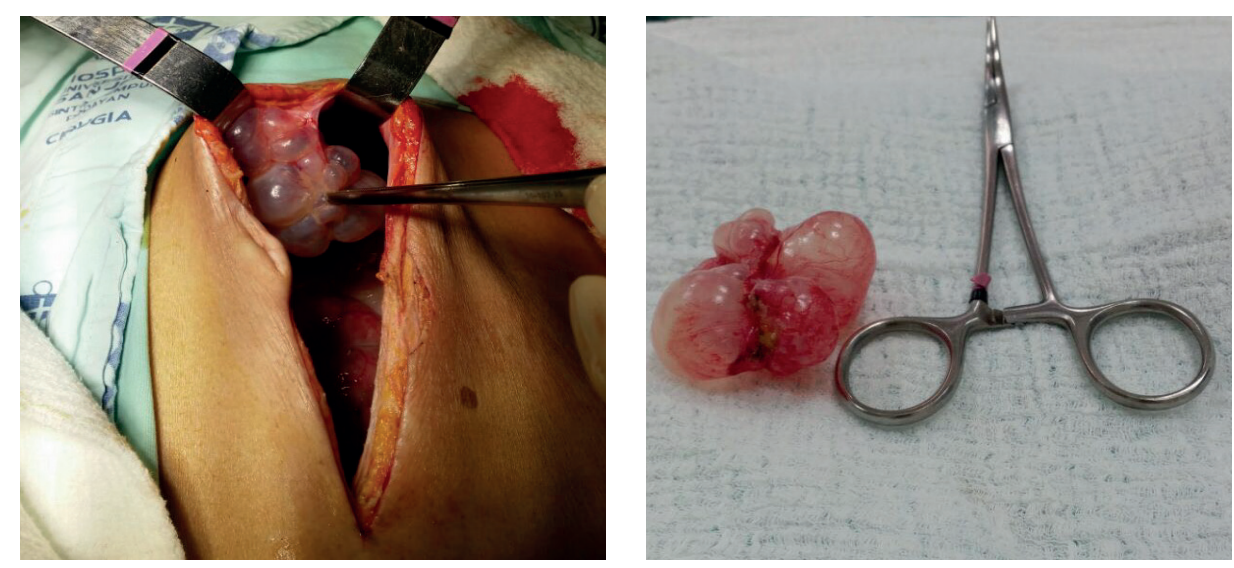

Figura 4. Quistes neumáticos adheridos al ligamento falciforme del hígado

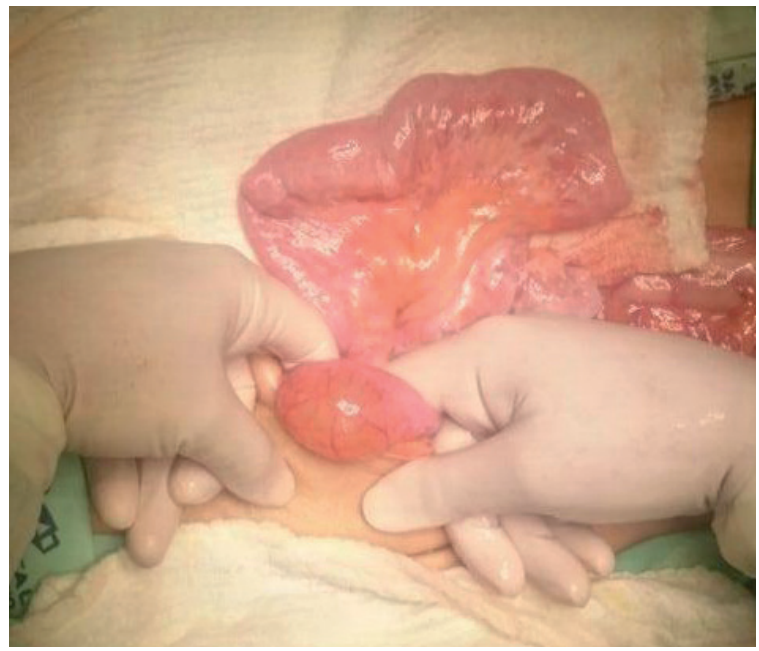

Figura 5. Quistes neumáticos en algunas asas intestinales delgadas

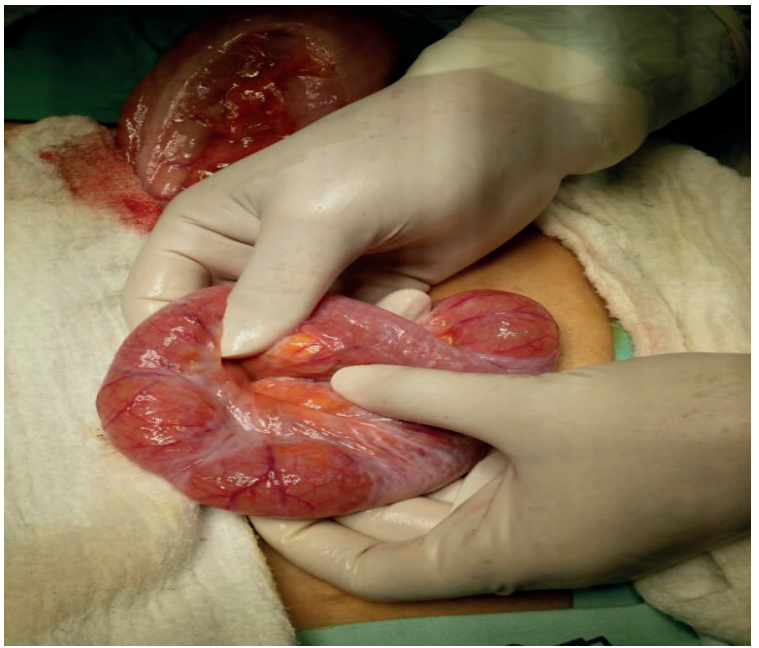

Figura 6. Zonas ovoides de serosa despulida en las asas intestinales, que sugieren ruptura espontánea de los quistes neumáticos intestinales. 


\section{Conclusiones}

- No todos los casos de neumoperitoneo son quirúrgicos. Aunque son raros, existen casos espontáneos o secundarios a neumatosis intestinal, por lo que este diagnóstico debe tenerse en cuenta en pacientes que consulten por neumoperitoneo sin signos de irritación peritoneal.

- En los casos de neumoperitoneo en pacientes estables, en quienes el diagnóstico etiológico no sea muy claro y se desee descartar perforación intestinal o isquemia, la laparoscopia diagnóstica es una buena opción para la exploración quirúrgica de estos pacientes y menos agresiva que la laparotomía.

- La neumatosis intestinal en pacientes con esclerosis sistémica es un marcador de mal pronóstico, y requiere el ajuste pertinente y temprano de la enfermedad de base.

\section{Cumplimiento de normas éticas}

Consentimiento informado: Este estudio es una revisión de la literatura científica y, como tal, no hay necesidad de un consentimiento informado. Se obtuvo aprobación por parte del Comité de Ética médica del hospital.

Declaración de conflicto de intereses: ninguno reportado por los autores.

Fuentes de financiación: recursos propios de los autores.

\section{Referencias}

I. Lee HS, Cho YW, Kim KJ, Lee JS, Lee SS, Yang SK. A simple score for predicting mortality in patients with pneumatosis intestinalis. Eur J Radiol. 2014;83:639-45. https://doi.org/IO.IOI6/j.ejrad.20I4.0I.003

2. Aziret M, Erdem H, Ülgen Y, Kahramanca S, Çetinkünar S, Bozkurt H, et al. The appearance of free-air in the abdomen with related pneumatosis cystoides intestinalis: Three case reports and review of the literature. Int
J Surg Case Rep. 20I4;5:909-I3. https://doi.org/IO.IOI6/j. ijscr.20I4.09.03I

3. Lombana J, Valencia A. Neumatosis quística intestinal neumoperitoneo: manejo conservador. Universitas Médica. 2005;46:59-6I.

4. Selles R, Zumarraga P, Ruiz J. Neumoperitoneo debido a neumatosis quística intestinal. Cir Esp. 2004;76:396-9. https://doi.org/IO.IOI6/Sooo9-739X(04)72402-I

5. Florez LE, García JF. Neumatosis quística intestinal. Rev Colomb Cir. 2003;18:225-9.

6. Karabuga T, Yoldas O, Ozsan I, Yıldırım UM, Aydin U. Diagnostic laparoscopy for pneumatosis intestinalis: To do or not to do? Am J Emerg Med. 2014;32:I555.eI-2. https://doi.org/Io.IOI6/j.ajem.20I4.04.042

7. Klimova K, Pérez M, Merino B, Gonzales C, Menchen $\mathrm{P}$. Pneumatosis cystoides intestinalis as an infrequent cause of chronic abdominal pain. Rev Gastroenterol Mex. 20I4;79:302-3. https://doi.org/IO.IOI6/j.rgmxen.20I4.II.003

8. Goldberg E, Thomas J. Pneumatosis intestinalis. Fecha de consulta: Io de abril de 20I6. Disponible en: http:// www.uptodate.com/contents/pneumatosis-intestinalis.

9. Millet A, Desfourneaux V, Jego P. Pneumatosis cystoides intestinalis in systemic sclerosis. Joint Bone Spine. 2008;75:367-8. https://doi.org/Io.IoI6/j.jbspin. 2007.08.004

Io. Hamada M, Kayashima M, Morai Y, Chi Masuda T, Urabe K, Furue M. Pneumatosis cystoides intestinalis with systemic sclerosis, limited type resulting in a poor prognosis. Am J Med Sci. 2006;332:I00-2. https://doi. org/I0.I097/0000044I-200608000-000II

II. Arikanoglu Z, Aygen E, Camci C, Akbulut S, Basbug $\mathrm{M}$, Dogru $\mathrm{O}$, et al. Pneumatosis cystoides intestinalis: A single center experience. World J Gastroenterol. 20I2;I8:453-7. https://doi.org/Io.3748/wjg.vI8.i5.453

I2. Zuluaga A, Mejía J, Uribe R, Gutiérrez C, Bustamante S, Vega M. Neumatosis intestinal: ¿una urgencia abdominal? Rev CES Med. 20I7;3I:IIO8. https://doi.org/Io.216I5/ cesmedicina.3I.I.II

13. Sugihara Y, Harada K, Ogawa H, Otsuka F, Okada H. Pneumatosis cystoides intestinalis. J Lung Health Dis. 20I8;2:24-7. https://doi.org/IO.29245/2689-99 9X/20I7/I.II2I

I4. Rachapalli V, Chaluvashetty SB. Neumatosis cystoides intestinalis. J Clin Diagn Res. 20I7;II:TJoI-TJo2. https:// doi.org/I0.7860/JCDR/2017/26197.10087 